\title{
Effects of food on physical and sleep complaints in children with ADHD: a randomised controlled pilot study
}

\author{
Lidy M. Pelsser • Klaas Frankena • Jan K. Buitelaar • \\ Nanda N. Rommelse
}

Received: 14 December 2009 / Accepted: 23 March 2010 /Published online: 17 April 2010

(C) The Author(s) 2010. This article is published with open access at Springerlink.com

\begin{abstract}
Attention deficit/hyperactivity disorder (ADHD), a common behavioural disorder in children, may be associated with comorbid physical and sleep complaints. Dietary intervention studies have shown convincing evidence of efficacy in reducing ADHD symptoms in children. In this pilot study, we investigated the effects of an elimination diet on physical and sleep complaints in children with ADHD. A group of 27 children (3.8-8.5 years old), who all met the Diagnostic and Statistical Manual of Mental Disorders, Fourth Edition criteria for ADHD, were assigned randomly to either a diet group (15/27) or a control group (12/27). The diet group followed a 5-week elimination diet; the control group adhered to their normal diet. Parents of both groups had to keep an extended diary and had to monitor the behaviour and the physical and sleep complaints of their child conscientiously. The primary endpoint was the clinical response, i.e. a decrease of physical and sleep complaints, at the end of the trial, based on parent ratings on a Physical Complaints Questionnaire. The number of physical and sleep complaints was significantly decreased in the diet group compared to the control
\end{abstract}

L. M. Pelsser $(\bowtie)$

ADHD Research Centre,

Liviuslaan 49,

5624 JE Eindhoven, The Netherlands

e-mail: 1mjpelsser@adhdresearchcentre.nl

K. Frankena

Quantitative Veterinary Epidemiology Group,

Wageningen University,

Wageningen, The Netherlands

J. K. Buitelaar • N. N. Rommelse

Radboud University Nijmegen Medical Centre and Karakter,

Child and Adolescent Psychiatry University Center Nijmegen,

Nijmegen, The Netherlands group ( $p<0.001)$, with a reduction in the diet group of $77 \%$ $(p<0.001$, effect size $=2.0)$ and in the control group of $17 \%$ $(p=0.08$, effect size $=0.2)$. Specific complaints that were significantly reduced were in three domains: headaches or bellyaches, unusual thirst or unusual perspiration, and sleep complaints. The reduction of complaints seemed to occur independently of the behavioural changes $(p=0.1)$. However, the power of this comparison was low. A positive correlation existed between the reduction of physical and behavioural symptoms $(p<0.01)$. The reduction did not differ between children with or without an atopic constitution $(p=0.7)$. An elimination diet may be an effective instrument to reduce physical complaints in children with ADHD, but more research is needed to determine the effects of food on (functional) somatic symptoms in children with and without ADHD. This trial was registered as an International Standard Randomised Controlled Trial, ISRCTN47247160.

Keywords ADHD - Children · Elimination diet . Physical complaints $\cdot$ Sleep problems

$\begin{array}{ll}\text { Abbreviations } \\ \text { ADHD } & \text { Attention deficit/hyperactivity disorder } \\ \text { OR } & \text { Odds ratio } \\ \text { PCQ } & \text { Physical Complaints Questionnaire } \\ \text { RCT } & \text { Randomised controlled trial }\end{array}$

\section{Introduction}

Attention deficit/hyperactivity disorder (ADHD) [1], one of the most common behavioural disorders in childhood, with symptoms of inattention, hyperactivity and impulsivity [18], often coexists with other problems, like oppositional 
defiant disorder, depression, anxiety, physical complaints (e.g. headache, eczema and diarrhoea) and sleep complaints $[9-11,13,14,16,21,22,27,29,35,39]$. The exact aetiological pathways of ADHD are still unknown: genetic risk factors including multiple genes (some of which are involved in the regulation of the immune system [30]) and environmental factors are involved.

To date, pharmacotherapy, combined with behavioural management, is the most effective treatment of ADHD [20]. Despite initial symptom improvement during this treatment, the follow-up study of the Multimodal Treatment Study of children with combined-type ADHD [24] showed that these children exhibit significant impairment in adolescence, implicating that innovative treatment approaches are needed [24]. Moreover, as treatment with psychostimulants like methylphenidate, with a duration of action of between 3 and $12 \mathrm{~h}$ [39], neither leads to resolve the behavioural problems in the early morning and in the evening nor resolves the comorbid physical complaints, it is worthwhile to investigate other treatments of ADHD and their effects on comorbid complaints.

One of these alternative treatment methods for ADHD may be an elimination diet. The effects of an elimination diet on ADHD have been investigated in several controlled studies $[5,9,13,22,31,34,36]$, showing a significant effect of a restricted elimination diet on symptoms of ADHD and establishing that there clearly is a diet behaviour connection $[2,6]$. Considering the comorbidity between ADHD and physical complaints (in one study, 20 out of 31 children with ADHD were reported to have at least two physical complaints [29]), one may speculate about a connection between food, ADHD and physical complaints. Given that (1) an elimination diet can significantly reduce ADHD symptoms [5, 9, 13, 22, 31, 34, 36], (2) the vast majority of children with ADHD suffers from co-occurring physical complaints $[9,13,22,29]$, (3) children with ADHD and extensive physical problems tend to respond less favourably to medication [3], (4) medication treatment does not solve the physical complaints or even causes some of these complaints [39] and (5) that a diet can have a positive effect on physical complaints in children and adults without ADHD [4, 41], it is timely to study the potentially beneficial effects an elimination diet may have on physical and sleep complaints in children with ADHD.

In four previous studies, the effect of an elimination diet on comorbid physical symptoms in children with ADHD has already been investigated, resulting both in a reduction of the behavioural as well as of the physical complaints [9, 13, 22, 29]. Most of the children participating in these studies were diagnosed with allergy or had an atopic constitution (being defined as having at least one parent or sibling with an allergic disease like asthma, eczema, hay fever or allergic rhinitis), thus limiting the extrapolation of findings to children with ADHD without allergies or an atopic constitution. Another limitation of these previous studies is that they did not report on whether or not the improvement of physical complaints coincided with improvements in ADHD symptoms. It is important for clinical health care, i.e. to predict the effects of an elimination diet, and for scientific reasons, i.e. to increase our knowledge about the aetiology of ADHD and physical complaints, to investigate whether reduction in ADHD symptoms and physical complaints go hand in hand when applying an elimination diet.

The current study aimed to examine these issues. We previously reported that an elimination diet had a statistically significant and clinically relevant effect on ADHD symptoms as reported by both parents and teachers, with effect sizes of 2.1 and 2.5, respectively [31]. The results on the endpoints concerning physical and sleep complaints will be presented in this paper. More specifically, we aimed to (1) examine whether physical and sleep complaints in children with ADHD could be diminished using an elimination diet, (2) investigate whether the effect of an elimination diet on physical and sleep complaints was limited to those children who clearly showed behavioural improvements to the elimination diet, and (3) investigate whether the effect of an elimination diet on physical and sleep complaints was restricted to children with an atopic constitution.

\section{Subjects and methods}

\section{Subjects}

Participants were selected from a sample of 79 Dutch children who were referred to the Dutch ADHD Research Centre in Eindhoven, specialised in scientific research on food and ADHD. Children were included if (1) they were between 3 and 8 years old; (2) they met the criteria for ADHD, as defined in the Diagnostic and Statistical Manual of Mental Disorders, Fourth Edition for ADHD Combined Type or Predominantly Hyperactive-Impulsive Type [1]; (3) their behavioural problems were present before the age of 4 or (4) they were medication naïve. Children were excluded if (1) they were diagnosed with Autism Spectrum Disorder or with ADHD Predominantly Inattentive Type [28], (2) they were pre- or dysmature at birth $[30,40]$ or (3) the mother had been smoking during pregnancy. A total of 43 children of the 79 failed to meet the criteria, and nine refused to participate. As a result, 27 children entered the study between January and July 2006 and were randomly allocated to the diet group (15) or the control group (12; Fig. 1). Twenty-four children, 13 in the diet group and 11 in the control group, completed the study. At the start of the 
Fig. 1 Flow diagram of subject participation throughout the day

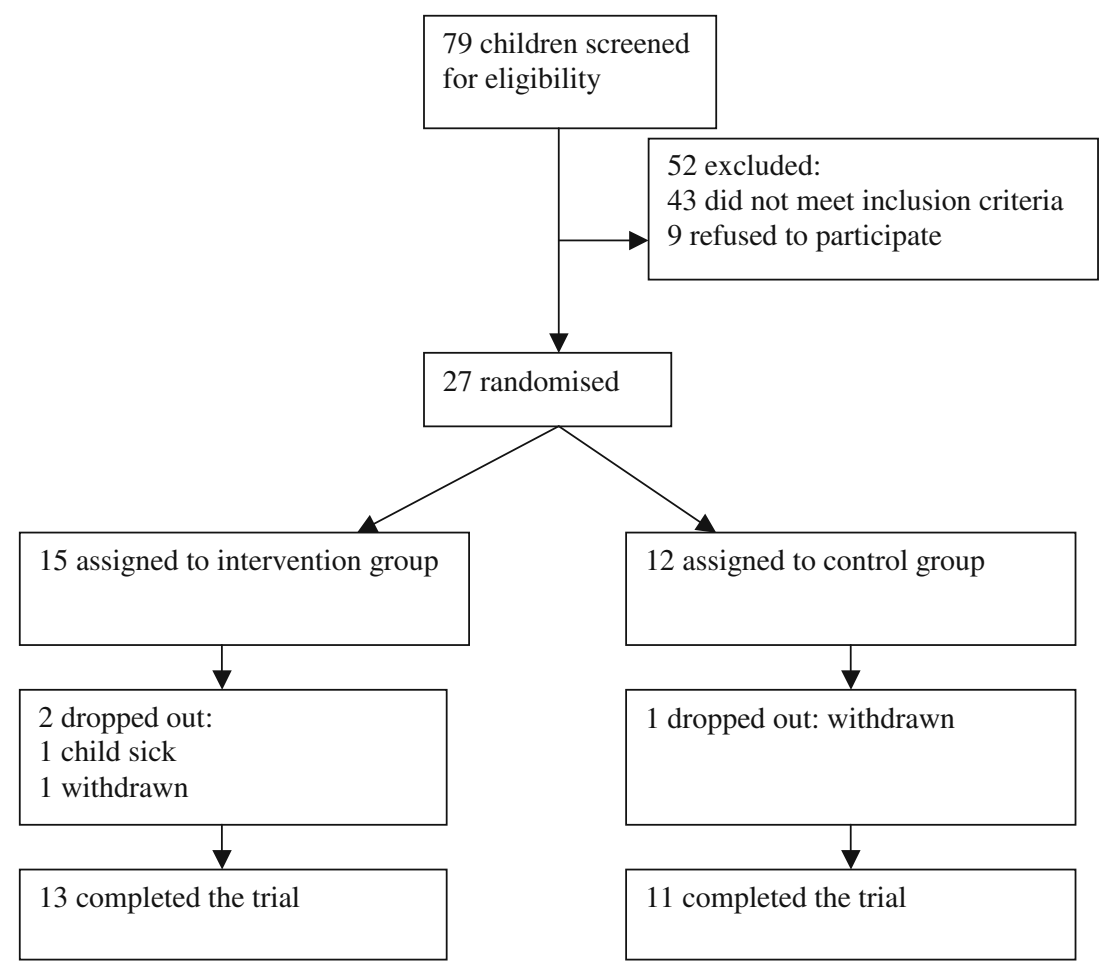

trial, there was no difference between the number of physical complaints or the severity of ADHD symptoms in diet group and control group (Table 1).

\section{Protocol}

The efficacy of an elimination diet on the reduction of physical and sleep complaints in children with ADHD was tested in this randomised controlled trial (RCT) by comparing outcomes within diet and control group, before and after intervention and between groups. Subjects were randomly allocated to one of the two groups by means of a sequence of numbered cards in sealed unmarked envelopes that were prepared by an independent paediatrician. Each card contained a reference to the group to which the child would be allocated, and for each allocation, an equal number of cards (20) were available. The envelopes were picked and opened by the parents in the presence of the researcher, and treatment was then dispensed in accordance to the allocation on the card.

All children started with a 2-week baseline diet in which they adhered to their normal diet; no foods were eliminated. Children who were already on a diet had to cease this diet at least 2 weeks before the start of the trial. During the baseline diet, the parents kept an extended diary and had to observe their child carefully, in order to assess the child's normal diet, his/her behaviour, physical complaints, sleep complaints and activities. There were two measurement

Table 1 Baseline characteristics of the 24 children who completed the interventions

\begin{tabular}{|c|c|c|c|}
\hline Characteristic & Diet group, $N=13$ & Control group, $N=11$ & Fisher exact $p$ value (two-sided) \\
\hline Boys & $10 / 13(76.9 \%)$ & $9 / 11(81.8 \%)$ & $>0.99$ \\
\hline Mean age (SD) & $6.3(1.6)$ & $6.2(1.7)$ & $0.91^{\mathrm{a}}$ \\
\hline Mean number of ADHD criteria (SD) & $14.4(2.0)$ & $13.7(2.1)$ & $0.44^{\mathrm{a}}$ \\
\hline Comorbid ODD & $12 / 13(80.0 \%)$ & $10 / 11(83.3 \%)$ & 0.60 \\
\hline Atopic constitution family & $9 / 13(69.2 \%)$ & $8 / 11(72.7 \%)$ & 0.99 \\
\hline Allergy diagnosed in child & $1 / 13(7.7 \%)$ & $2 / 11(18.2 \%)$ & 0.58 \\
\hline On dietary restriction & $0 / 13(0 \%)$ & $1 / 11(9.1 \%)$ & 0.46 \\
\hline Mean number of physical problems (SD) & $3.0(1.4)$ & $2.8(2.2)$ & $0.81^{\mathrm{a}}$ \\
\hline Sleep complaints & $5 / 13(38.5 \%)$ & $5 / 11(45.5 \%)$ & $>0.99$ \\
\hline
\end{tabular}

$N$ number of participants

${ }^{\text {a }}$ Student's $t$ test 
points: at the end of the baseline diet and at the end of the elimination diet (diet group) or the control period (control group).

The diet group started, after the baseline diet and the first assessment, with the elimination diet, which had to be followed for a period of 5 weeks. The elimination diet was based on a few foods diet, as described by Hill and Taylor in their basic algorithm for treatment of ADHD [19]. The rationale behind the few foods diet was the assumption that children might present with ADHD symptoms after eating any kind of foods. Therefore, the diet consisted only of a limited number of hypoallergenic foods, like rice, turkey, lamb, a range of vegetables (lettuce, carrots, cauliflower, cabbage and beet), pears and water. All other foods were prohibited, but vegetables, fruits, rice and meat were allowed every day, in normal doses. Calcium was supplied daily via non-dairy rice drink with added calcium; children were not at risk for nutrient deficiencies. This few foods diet was complemented with specific foods like potatoes, fruits, corn and wheat, to be eaten on days and in doses stated in advance according to a compulsory intake schedule $[9,29,31]$. As a result of this strategy, an elimination diet as comprehensive as possible could be composed for each individual child, thus making the intervention less incriminating for child and parents. If there was no improvement by the end of the second week, the diet was restricted and gradually limited to the few foods diet $[9,29,31]$. The second measurement point occurred at the end of the elimination diet.

The control group continued, after the first assessment, their baseline diet, i.e. their normal diet in which no foods were excluded, for a period of 5 weeks. Unfortunately, in dietary studies using a very restricted diet, it is not possible to create a reliable placebo diet, thus impeding a placebo-controlled trial. Therefore, this study is an RCT, which is often used in studies when no placebo is available, such as studies into the effects of cognitive behaviour therapy, eczema or other medical intervention trials $[32,37,38,41,43,45]$. As it is conceivable that the child's behaviour and somatic complaints might improve because of the special attention which parents have to give to their child in order to fill in the diary correctly, parents of children in the control group also had to keep an extended diary and had to monitor the behaviour and the physical and sleep complaints of their child conscientiously. The second measurement point was at the end of the control period.

At the start of the trial, the parents of the control group were informed that they could start with the elimination diet immediately after the last assessment, if they wished so. Parents were given verbal and written information about the study, and written informed parental consent was obtained before randomisation.
Measures

Physical and sleep complaints were measured using the Physical Complaints Questionnaire (PCQ) [28, 29]. This questionnaire consisted of 36 questions, of which 18 items were relevant with respect to specific physical and sleep complaints. Items were rated on a four-point scale, concerning the problems during the past week: problems which occurred every day (3), several times a week (2), once a week (1) or less than once a week (0). The questionnaire had to be filled in by the parents twice, e.g. before and after the elimination diet or control period. The physical complaints concerned 16 items and were subtyped into seven domains: (1) pain (headaches, abdominal pains and growing pains), (2) unusual thirst or unusual perspiration, (3) eczema, (4) asthma or persisting cold (rhinitis), (5) skin problems (blotches in the face, red ears, red-edged mouth or bags under the eyes), (6) tiredness and (7) gastrointestinal problems (diarrhoea, constipation and flatulence). Two of the 18 questions concerned sleep complaints, i.e. problems with sleeping in (sleep initiation or sleep onset) and sleeping on (sleep maintenance). A domain was considered to be present when rated 2 (several times a week) or 3 (every day) for at least one of the items within that domain. A problem was considered to be absent when the score was 0 or 1 for all items within that domain.

\section{Statistical analysis}

Main endpoints were the parent ratings on the PCQ at the end of the RCT to establish the effect of the intervention on physical and sleep complaints. Differences in averages within groups (effect size), before and after the trial, were tested by Student's $t$ test and expressed by Cohen's $d$, a standardised measure of the effect size with an effect size of 0.2 indicative of a small effect and 0.8 of a large effect. Differences in average number of complaints between groups, at the end of the trial, were analysed using linear regression, including the number of complaints at the start of the trial as covariate.

Differences in presence/absence of complaints between groups, at the end of the trial, were analysed using exact logistic regression, because the endpoints were binary. PCQ ratings at the start of the study were included as covariate. Here, the effect of intervention was expressed in terms of odds ratios (OR) and their $p$ values.

After finishing the RCT, all children in the control group $(N=11)$ also completed the elimination diet, resulting in 24 children in total who underwent the elimination diet, i.e. 13 children from the diet group, during the RCT, and 11 children from the control group, following the RCT. The secondary endpoints, analysed using linear and exact logistic regression, and calculated in all 24 children who 
completed the diet, were (1) the effects of the elimination diet on physical and sleep complaints in children who showed ADHD symptom reduction of $50 \%$ or more after following the elimination diet, i.e. responders, and in children who showed less than 50\% ADHD symptom reduction, the non-responders [31] and (2) the effects of the elimination diet on physical and sleep complaints in children with and without an atopic constitution.

Spearman rank correlation coefficients were calculated to study the improvement of physical complaints and ADHD core symptoms after having followed the diet.

STATA 10 was used for all statistical analyses. Effects were tested at $p=0.05$.

\section{Results}

Effect of the intervention on physical and sleep complaints in diet group and control group

The results of the intervention on physical and sleep complaints in both groups are shown in Table 2 and Fig. 2. The total number of complaints in the diet group was 44 (average, 3.4 per child) at the start of the trial and ten complaints (average, 0.8) at the end of the trial, a reduction of $77 \%(p<0.001)$, with a standardised effect size (Cohen's $d$ ) of 2.0 (Table 3). In the control group, 36 complaints (average, 3.3) were reported at the start of the trial and 30 (average, 2.7) at the end of the trial, a reduction of $17 \%(p=0.08)$, with an effect size of 0.2 .

Using linear regression and taking the initial number of complaints into account, the difference in average number of complaints at the end of the trial between the diet and control group equalled 2.04 (95\% confidence interval (CI) $1.14-2.94, p<0.001$, residuals being normally distributed, $p$ value of Shapiro-Wilk test equals 0.55 ).

In three domains ((1) headaches or bellyaches $(\mathrm{OR}=$ 13.25), (2) unusual thirst or unusual perspiration $(\mathrm{OR}=$ 10.04) and (3) sleep complaints $(\mathrm{OR}=11.77))$, the complaints were significantly less reduced in the control group than in the diet group $(p<0.05)$.

Effect of the elimination diet on physical and sleep complaints in children with and without ADHD symptom reduction

This effect was calculated in all 24 children who followed the elimination diet, 13 children of the diet group and 11 children of the control group following the RCT. Of these children, 20/24 belonged to the responders, 11/13 children of the diet group and 9/11 children of the control group. The responders, having to show a minimal ADHD symptom reduction of $50 \%$, showed an average reduction on the ADHD rating scale of $69.4 \%$ (effect size, 2.1), according to the parent ratings, and an ADHD symptom reduction of $70.6 \%$ (effect size, 2.5), according to the teacher ratings.

Before following the diet, there was an average of 3.2 physical and sleep complaints per child in the responder group and 2.5 in the non-responder group. After the diet, these averages were 0.9 and 1.5 , respectively. In the responder group, there was a significant reduction of physical complaints $(p<0.001)$; in the non-responder group, the reduction was not significant $(p=0.35)$, with standardised effect sizes of 1.4 and 0.8 , respectively.

Table 2 Effect of the interventions on physical and sleep complaints

\begin{tabular}{|c|c|c|c|c|c|c|}
\hline & \multicolumn{2}{|c|}{ Diet group, $N=13$} & \multicolumn{2}{|c|}{ Control group, $N=11$} & \multirow[t]{2}{*}{ Exact OR } & \multirow[t]{2}{*}{$p$ value $\mathrm{OR}$} \\
\hline & $\begin{array}{l}\text { Start trial } \\
N \text { children } \\
\% \text { children }\end{array}$ & $\begin{array}{l}\text { End trial } \\
N \text { children } \\
\% \text { children }\end{array}$ & $\begin{array}{l}\text { Start trial } \\
N \text { children } \\
\% \text { children }\end{array}$ & $\begin{array}{l}\text { End trial } \\
N \text { children } \\
\% \text { children }\end{array}$ & & \\
\hline Headaches, abdominal pains, growing pains & $9(69 \%)$ & $1(8 \%)$ & $9(82 \%)$ & $6(54 \%)$ & $13.25^{\mathrm{a}}$ & 0.05 \\
\hline Unusual thirst, unusual perspiration & $10(77 \%)$ & $0(0 \%)$ & $6(54 \%)$ & $4(27 \%)$ & 10.04 & 0.05 \\
\hline Eczema & $1(8 \%)$ & $0(0 \%)$ & $5(45 \%)$ & $5(45 \%)$ & 5.00 & 0.33 \\
\hline Asthma, rhinitis & $2(15 \%)$ & $1(8 \%)$ & $2(18 \%)$ & $1(9 \%)$ & 1.00 & 0.99 \\
\hline $\begin{array}{l}\text { Blotches in face, red ears, red-edged mouth, } \\
\text { bags under eyes }\end{array}$ & $2(15 \%)$ & $1(8 \%)$ & $3(27 \%)$ & $3(27 \%)$ & 1.50 & 0.80 \\
\hline Tiredness & $9(69 \%)$ & $4(31 \%)$ & $3(27 \%)$ & $3(27 \%)$ & 3.58 & 0.32 \\
\hline Diarrhoea, constipation, flatulence & $6(46 \%)$ & $2(15 \%)$ & $3(27 \%)$ & $3(27 \%)$ & 4.66 & 0.24 \\
\hline Problems with sleeping in or sleeping on & $5(38 \%)$ & $1(8 \%)$ & $5(45 \%)$ & $5(45 \%)$ & 11.77 & 0.05 \\
\hline $\begin{array}{l}\text { Total number of complaints, including } \\
\text { sleep complaints }\end{array}$ & 44 & 10 & 36 & 30 & $16.20^{\mathrm{b}}$ & 0.001 \\
\hline
\end{tabular}

${ }^{a}$ The odds of having complaints at the end of the trial is 13.25 -fold higher in the control group compared to the diet group

${ }^{\mathrm{b}}$ Based on 80 initial complaints 
Fig. 2 Physical and sleep complaints in the diet group and the control group at start and at endpoint. Pain: headaches, abdominal pains or growing pains. Thirst: unusual thirst or unusual perspiration. Skin: eczema. Asthma: asthma or persisting cold (rhinitis). Blotch: blotches in the face, red ears, red-edged mouth or bags under the eyes. Tired: tiredness. Bowel: diarrhoea, constipation or flatulence. Sleep: sleeping in or sleeping on
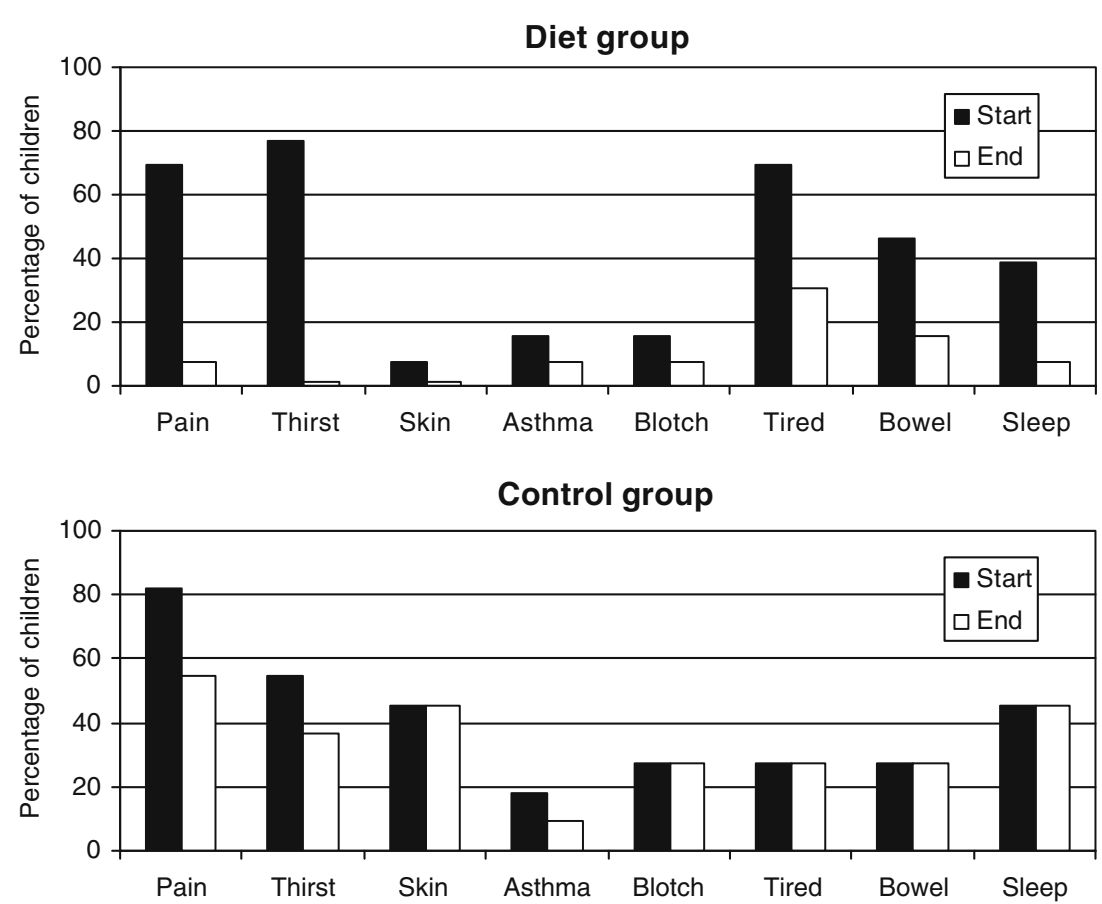

Linear regression, including the initial number of complaints as covariate, revealed a difference in average number of complaints between the responder and nonresponder group of 0.82 ( $p$ value 0.10 , residuals normally distributed, $p$ value of Shapiro-Wilk test equals 0.62).

The correlation between the total number of physical and sleep complaints and the total number of ADHD criteria on the ADHD rating scale, before and after the diet, was calculated using Spearman rank correlation coefficients. Spearman's rho was $0.54(p<0.01)$, indicating there was a positive correlation between the reduction of the physical and the behavioural symptoms.

Statistical analyses to investigate the difference in effect of the elimination diet on the specific physical domains in responders and non-responders could not be performed as there were only four non-responders.
Effect of the elimination diet on physical and sleep complaints in children with and without an atopic constitution

This effect was calculated in all 24 children who followed the elimination diet. An atopic constitution, i.e. having at least one parent or sibling with allergic complaints like asthma, eczema, hay fever or allergic rhinitis, was present in $17 / 24(70.8 \%)$ children, equally divided over the diet group $(9 / 13,69.2 \%)$ and the control group $(8 / 11,72.7 \%$; Fisher's exact test, $p=0.99$ ). At the start of the trial, an average of 3.5 complaints per child was observed in the atopic children and an average of 2.0 per child in the nonatopic group. After the diet, these averages were 1.2 and 0.6 , respectively. In the atopic as well as in the non-atopic children, there was a significant reduction of physical

Table 3 Average number of physical complaints, including sleep complaints, per child per intervention group at start and at endpoint

Average no. of complaints

\begin{tabular}{llll}
\hline \multirow{2}{*}{ Start } & End & \multicolumn{2}{l}{ Start minus end } \\
\cline { 3 - 4 } & & Difference $(95 \% \mathrm{CI})$ & Effect size $^{\mathrm{a}}(\% \mathrm{SR}), p$ value \\
\hline $3.4^{2}$ & 0.8 & $2.6(1.8$ to 3.4$)$ & $2.0(77.3) p=0.001$ \\
$3.3^{\mathrm{b}}$ & 2.7 & $0.6(-0.1$ to 1.2$)$ & $0.2(16.7) p=0.08$ \\
\hline
\end{tabular}

\begin{tabular}{ll}
\hline Diet group $(N=13)$ & $3.4^{2}$ \\
Control group $(N=11)$ & $3.3^{\mathrm{b}}$ \\
\hline$S R$ scale reduction & \\
${ }^{\mathrm{a}}$ Effect size start-end, Cohen's $d$ & \\
${ }^{\mathrm{b}}$ Difference at start $p=0.89$ (Student's $t$ test)
\end{tabular}


complaints, ( $p$ values $<0.001$ and 0.04 , respectively) with standardised effect sizes of 1.5 and 1.1, respectively.

Although the reduction within both groups was significant, linear regression did not show a significant difference between the atopic and non-atopic group at the end of the diet while adjusting for the initial number of complaints (difference equals 0.18 complaints, $p=0.70$, residuals normally distributed, $p$ value of Shapiro-Wilk test equals 0.73).

Complaint specific analyses could not be performed due to the low number of non-atopic children $(n=7)$.

\section{Discussion}

Physical complaints, such as headache, bellyache, tiredness, eczema and sleep complaints, are common comorbid problems in children with ADHD [10, 11, 16, 21, 27, 35, 39], with a prevalence of sleep complaints up to 50\% [39]. In contrast to comorbid psychiatric conditions, relatively little is known on the comorbidity of ADHD and physical complaints. In this study, we examined whether physical and sleep complaints in 24 children with ADHD were improved by an elimination diet using a randomised controlled design. We previously described that the diet significantly reduced the ADHD symptoms in this group of patients [31]. In the current study, in which the subjects were not preselected for somatic symptoms, 23/24 (96\%) children had one or more physical complaints, indicating that comorbidity between ADHD and physical complaints is high, thus underlining the importance of studying physical complaints in ADHD.

The results of this pilot study should be interpreted in the light of several limitations. First, in this study, a very restricted elimination diet was used, thus making it impossible to compose a reliable placebo diet. Furthermore, parents had to be aware of the intervention and had to pay attention to what the child should eat. Therefore, we had to choose for an open RCT. Although a blinded RCT should be given preference to, open RCTs are commonly used and accepted when blinding is difficult and when no placebo is available, e.g. in studies into the effects of cognitive behaviour therapy, eczema, obesity, autism or other medical intervention trials $[7,12,15,32,37,38,41,43,45]$. Also, the well known and highly cited Multimodal Treatment Study of Children with ADHD, the MTA-study, was not blinded [26]. To compensate for the absence of a placebo diet, the parents in the control group, like the parents in the diet group, had to monitor and to observe their child intensively, writing down the behaviour and the physical and sleep complaints of their child conscientiously in a diary. It is conceivable that the child's behaviour and somatic complaints might improve because of the special attention which parents had to pay to their child. In our study, the reduction of the total numbers of complaints in the diet group $(77 \%)$ was 4.6 -fold compared to the reduction in the control group $(17 \% ; p=0.001)$, indicating that the effect of an increase of attention may be small, when compared to the effect of an elimination diet. Second, the trial lasted only 5 weeks, which is a short period of time. Follow-up studies should include a follow-up period of at least 1 year. Finally, the sample size of the study was relatively small; consequently, the data reported here should be considered exploratory. Nevertheless, due to the considerable effect sizes in this study, statistically significant differences between diet and control were obtained.

The effect of the intervention on physical and sleep complaints did not differ significantly between children who did or did not show ADHD symptom reduction after following the diet. The adjusted difference between both groups amounted to $0.82(p=0.10)$, suggesting the diet is equally effective in reducing physical complaints in responders and non-responders. However, the power of this analysis is low (0.26), as the non-responder group consisted of four children only. Correlation analyses revealed that $\mathrm{ADHD}$ symptom reduction and the reduction of physical complaints were correlated significantly. We hypothesise, considering the effect size of an elimination diet on both ADHD and physical complaints, that there may be a common underlying mechanism for both conditions. This mechanism may be a hypersensitivity reaction to food, which could be an etiological factor of both conditions [30]. This hypersensitivity mechanism might either be allergic, i.e. related to the induction of $\operatorname{IgE}$ or IgG antibodies or of a cell-mediated response [30], or not allergic, i.e. related to a toxic or pharmacologic mechanism. When there is no effect of an elimination diet on one or more of the complaints, other etiological mechanisms are likely and should be considered.

In this study, $71 \%$ of the ADHD children had an atopic constitution. This high prevalence may be related to the possibility that parents acquainted with allergic disorders are more willing to let their child follow an elimination diet than parents unfamiliar with allergies. On the other hand, atopy is a widespread condition, found in many children. A UK study reported that $39 \%$ of children in the UK had been diagnosed with one or more atopic conditions [17], and positive skin prick tests to at least one allergen was found in $63.7 \%$ of urban children [23]. Our study shows that in atopic and in non-atopic children, the number of physical and sleep complaints did not differ significantly before $(p=$ $0.081)$ as well as after $(p=0.32)$ the elimination diet. We did find, although not statistically significant, that at the start of the trial more physical complaints were reported in atopic children (average, 3.5 per child) than in non-atopic children (average, 2.0 per child). The results of this study indicate 
that the presence of an atopic constitution is not a moderator of the effect of an elimination diet on physical complaints and sleep complaints in children with ADHD, but do suggest atopy is an important condition co-occurring with ADHD.

The subjects in our study were young, but children of 4 years and older are generally expected to be able to tell that it hurts and where it hurts. Therefore, headache, abdominal pains and pain in the legs or arms (growing pains) are probably reliably reported. However, restless legs or breathing difficulties may be more difficult for a child to describe, so it may be conceivable that the number of physical complaints is underestimated. We would like to emphasise that the sleep complaints were reported by the parents, not by the child. These complaints are generally well visible to the parents and have a large impact on family life.

As ADHD has an increased association with sleeprelated movement disorders such as restless legs syndrome [44], the relationship between food, ADHD and sleep complaints should be investigated more thoroughly in follow-up studies.

Although we do not know the mechanisms in which an elimination diet exerts its effects on physical and sleep complaints in ADHD, our findings indicate that the results of this study may be important for children with physical complaints or sleep complaints and ADHD. They even may be important for children with physical conditions without ADHD [4, 8, 25, 42] and for children with functional somatic symptoms, as these are common health complaints in 5-7-year-old children [33].

More research on the effects of foods and on the underlying mechanism is advised to investigate whether children with ADHD and co-occurring physical complaints may represent a specific ADHD subgroup. We hypothesise that there may be a common underlying genetic mechanism contributing to both medical conditions, comparable to the mechanism found by Campbell et al., in children with cooccurring autism and gastrointestinal conditions [8]. Consequently, the further unravelling of the genetic architecture of ADHD is very important to identify a common genetic pattern or genetic vulnerability in children with ADHD and physical complaints. Also, it is important to segregate between non-allergic or allergic mechanisms involved. This includes analysis of the role of $\operatorname{IgE}$ and $\operatorname{IgG}$ antibodies being specific for the food and the possible involvement of $\mathrm{T}$ cell-mediated hypersensitivity.

In studies specifically asking for physical complaints in children with ADHD, it turns out that comorbidity is high $[9,13,22,29]$. This high comorbidity between physical symptoms and ADHD does not reflect clinical practice, which may be due to the fact that in children with ADHD, it is not current practice to ask for physical complaints specifically. A general question like 'are there any physical complaints' may not be sufficient, generating too little information. Many of the physical symptoms investigated in this trial would not have been mentioned by the parents if we had not asked for them.

Because diets are not without its limitations (socially handicapping, putting a strain on the whole family), they should only be applied after responsiveness has been individually and carefully tested by means of an elimination diet, supervised and administered by trained staff [34]. If a child following the diet shows beneficial behavioural or physical effects, sequential introduction of foods is necessary to identify the incriminated foods $[9,13]$, so that the eventual diet of the child will be as comprehensive as possible. If a child who responds favourably to the diet will not proceed with this provocation period and returns to its usual diet, consequently, the problems are likely to return.

Further controlled studies are needed to verify the efficacy of an elimination diet in children with physical complaints and to provide a feasible algorithm for treatment, especially for children with behavioural or physical complaints triggered by foods. We will pursue this issue in a large $(N=100)$ sample of ADHD children using an RCT (the Impact of Nutrition on Children with ADHD study) currently underway, the protocol of which can be found on the website of The Lancet (http://www.thelancet.com/ protocol-reviews/06PRT-7719).

\section{Clinical implications and conclusion}

Our study shows that hypersensitivity to food may play an etiologic role in physical and sleep complaints in children with ADHD and suggests that an elimination diet may be a valuable tool to manage these problems in ADHD children. As functional somatic symptoms are common health complaints in 5-7-year-old children [33], the results of this study may be important for all children. Still, the sample size was small, and we cannot rule out expectation effects. Therefore, more research is needed to determine the effects of food on physical and sleep complaints in children with and without ADHD.

Acknowledgements We acknowledge the support for this study by the Foundation for Children's Welfare Stamps Netherlands, Foundation Nuts Ohra, Matty Brand Foundation and the Foundation of Child and Behaviour. The funding sources had no role in the study design, data collection, analysis or interpretation of the data and had no input into the writing of the report or in the decision to submit for publication. The authors' responsibilities were as follows: LP (Principal Investigator) designed the trial; KF performed the statistical analyses; LP, KF, JB and NR interpreted the data and wrote the manuscript. All authors critically revised the manuscript and approved the final report. All authors declare that they have no financial or commercial conflicts of interest. 
Open Access This article is distributed under the terms of the Creative Commons Attribution Noncommercial License which permits any noncommercial use, distribution, and reproduction in any medium, provided the original author(s) and source are credited.

\section{References}

1. American Psychiatric Association (1994) Diagnostic and statistical manual of mental disorders, 4th edn. APA, Washington DC

2. Arnold LE (1999) Treatment alternatives for ADHD. J Atten Disord 3:30-48

3. Barkley RA (1995) Taking charge of ADHD. Guilford, New York

4. Barros R, Moreira A, Fonseca J et al (2008) Adherence to the Mediterranean diet and fresh fruit intake are associated with improved asthma control. Allergy 63:917-923

5. Boris M, Mandel FS (1994) Food and additives are common causes of the attention deficit hyperactive disorder in children. Allergy 72:462-468

6. Breakey J (1997) Review article: the role of diet and behaviour in childhood. J Paediatr Child Health 33:190-194

7. Burgess IF, Brunton ER, Burgess NA (2010) Clinical trial showing superiority of a coconut and anise spray over permethrin $0.43 \%$ lotion for head louse infestation, ISRCTN96469780. Eur J Pediatr 169:55-62

8. Campbell DB, Buie TM, Winter H et al (2009) Distinct genetic risk based on association of MET in families with co-occurring autism and gastrointestinal conditions. Pediatrics 123:1018-1024

9. Carter CM, Urbanowicz M, Hemsley R et al (1993) Effects of a few food diet in attention deficit disorder. Arch Dis Child 69:564568

10. Cortese S, Faraone SV, Konofal E, Lecendreux M (2009) Sleep in children with attention-deficit/hyperactivity disorder: meta-analysis of subjective and objective studies. J Am Acad Child Adolesc Psychiatry 48:894-908

11. Cuffe SP, Moore CG, McKeown R (2009) ADHD and health services utilization in the national health interview survey. $\mathrm{J}$ Atten Disord 12:330-340

12. Dawson G, Rogers S, Munson J et al (2009) Randomized, controlled trial of an intervention for toddlers with autism: the early start Denver model. Pediatrics 125:e17-e23

13. Egger J, Carter CM, Graham PJ et al (1985) Controlled trial of oligo-antigenic treatment in the hyperkinetic syndrome. Lancet $1: 540-545$

14. Elia J, Ambrosini P, Berrettini W (2008) ADHD characteristics: I. Concurrent co-morbidity patterns in children $\&$ adolescents. Child Adolesc Psychiatry Ment Health 2:15

15. Garipağaoğlu M, Sahip Y, Darendeliler F et al (2009) Familybased group treatment versus individual treatment in the management of childhood obesity: randomized, prospective clinical trial. Eur J Pediatr 168:1091-1099

16. Ghanizadeh A, Moaiedy F, Imanieh MH et al (2008) Psychiatric disorders and family functioning in children and adolescents with functional abdominal pain syndrome. J Gastroenterol Hepatol 23:1132-1136

17. Gupta R, Sheikh A, Strachan DP, Anderson HR (2004) Burden of allergic disease in the UK: secondary analyses of national databases. Clin Exp Allergy 34:520-526

18. Hill P (2005) Attention deficit/hyperactivity disorder. Arch Dis Child 90:i1

19. Hill P, Taylor E (2001) An auditable protocol for treating attention deficit/hyperactivity disorder. Arch Dis Child 84:404-409

20. Jensen PS, Hinshaw SP, Swanson JM et al (2001) Findings from the NIMH Multimodal Treatment Study of ADHD [MTA]: implications and applications for primary care providers. J Dev Behav Pediatr 22:60-73

21. Kaplan BJ, McNicol J, Conte RA, Moghadam HK (1987) Physical signs and symptoms in preschool-age hyperactive and normal children. J Dev Behav Pediatr 8:305-310

22. Kaplan BJ, McNicol J, Conte RA, Moghadam HK (1989) Dietary replacement in preschool-aged hyperactive children. Pediatrics 83:7-17

23. Majkowska-Wojciechowska B, Pełka J, Korzon L et al (2007) Prevalence of allergy, patterns of allergic sensitization and allergy risk factors in rural and urban children. Allergy 2:1044-1050

24. Molina BS, Hinshaw SP, Swanson JM, MTA Cooperative Group et al (2009) The MTA at 8 years: prospective follow-up of children treated for combined-type ADHD in a multisite study. J Am Acad Child Adolesc Psychiatry 48:484-500

25. Association Mieux prescrire (2009) Irritable bowel syndrome: a mild disorder; purely symptomatic treatment. Prescrire Int 18:7579

26. The MTA Cooperative Group (1999) A 14-month randomized clinical trial of treatment strategies for attention-deficit/hyperactivity disorder. Multimodal treatment study of children with ADHD. Arch Gen Psychiatry 56:1073-1086

27. Owens JA (2009) A clinical overview of sleep and attentiondeficit/hyperactivity disorder in children and adolescents. J Can Acad Child Adolesc Psychiatry 18:92-102

28. Pelsser LM (2003) De invloed van voeding op hyperactief gedrag bij kinderen met ADHD. In Dutch. [The influence of food on hyperactive behaviour in children with ADHD]. Kind en Adolescent 1:4-16

29. Pelsser LM, Buitelaar JK (2002) Gunstige invloed van een standaardeliminatiedieet op het gedrag van jonge kinderen met aandachtstekort-hyperactiviteitsstoornis [ADHD], een verkennend onderzoek. In Dutch. [Favourable effects of an elimination diet on the behaviour of children with ADHD]. Ned Tijdschr Geneeskd 146:2543-2547

30. Pelsser LM, Buitelaar JK, Savelkoul HF (2009) ADHD as a [non] allergic hypersensitivity disorder: a hypothesis. Pediatr Allergy Immunol 20:107-112

31. Pelsser LM, Frankena K, Toorman J et al (2009) A randomised controlled trial into the effects of food on ADHD. Eur Child Adolesc Psychiatry 18:12-19

32. Prins JB, Bleijenberg G, Bazelmans E et al (2001) Cognitive behaviour therapy for chronic fatigue syndrome: a multicentre randomised controlled trial. Lancet 357:841-847

33. Rask CU, Olsen EM, Elberling H et al (2000) Functional somatic symptoms and associated impairment in 5-7-year-old children: the Copenhagen Child Cohort 2000. Eur J Epidemiol 24:625-634

34. Schmidt MH, Mocks P, Lay B et al (1997) Does oligoantigenic diet influence hyperactive/conduct-disordered children, a controlled trial. Eur Child Adolesc Psychiatry 6:88-95

35. Schmitt J, Romanos M, Schmitt NM et al (2009) Atopic eczema and attention-deficit/hyperactivity disorder in a population-based sample of children and adolescents. JAMA 18:724-726

36. Schulte-Korne G, Deimel W, Gutenbrunner C et al (1996) Effect of an oligo-antigen diet on the behaviour of hyperkinetic children. Z Kinder Jugendpsychiatr Psychother 24:176-183

37. Smithuis F, Kyaw MK, Phe O et al (2006) Efficacy and effectiveness of dihydroartemisinin-piperaquine versus artesunatemefloquine in falciparum malaria: an open-label randomised comparison. Lancet 367:2075-2085

38. Staab D, Diepgen TL, Fartasch M et al (2006) Age related, structured educational programmes for the management of atopic dermatitis in children and adolescents: multicentre, randomised controlled trial. Br Med J 332:933-938

39. Steer CR (2005) Managing attention deficit/hyperactivity disorder: unmet needs and future directions. Arch Dis Child 90:19-25 
40. Strang-Karlsson S, Räikkönen K, Pesonen AK et al (2008) Very low birth weight and behavioral symptoms of attention deficit hyperactivity disorder in young adulthood: the Helsinki study of very-low-birth-weight adults. Am J Psychiatry 165:1345-1353

41. Stulemeijer M, de Jong LW, Fiselier TJ et al (2005) Cognitive behaviour therapy for adolescents with chronic fatigue syndrome: randomised controlled trial. Br Med J 330:14-19

42. Sun-Edelstein C, Mauskop A (2009) Foods and supplements in the management of migraine headaches. Clin J Pain 25:446-452

43. van Wijck AJ, Opstelten W, Moons KG et al (2006) The PINE study of epidural steroids and local anaesthetics to prevent postherpetic neuralgia: a randomised controlled trial. Lancet 367:219-224

44. Walters AS, Silvestri R, Zucconi M et al (2008) Review of the possible relationship and hypothetical links between attention deficit hyperactivity disorder (ADHD) and the simple sleep related movement disorders, parasomnias, hypersomnias, and circadian rhythm disorders. J Clin Sleep Med 4:591-600

45. Wilson DC, Cairns P, Halliday HL et al (1997) Randomised controlled trial of an aggressive nutritional regimen in sick very low birthweight infants. Arch Dis Child Fetal Neonatal Ed 77:F4F11 